Pesq. Vet. Bras. 37(8):866-870, agosto 2017

DOI: $10.1590 / \mathrm{S} 0100-736 \mathrm{X} 2017000800014$

\title{
Estudo clínico e epidemiológico em cães com fraturas e luxações vertebrais toracolombares ${ }^{1}$
}

\author{
Bruno M. Araújo ${ }^{2 *}$, Thaíza H.T. Fernandes², Durval Baraúna Junior², Marília de A. \\ Bonelli², Marcela M. de A. Amorim² e Eduardo A. Tudury $^{2}$
}

\begin{abstract}
Araújo B.M., Fernandes T.H.T., Baraúna Junior D., Bonelli M.A., Amorim M.M.A. \& Tudury E.A. 2017. [Clinical and epidemiological study in dogs with thoracolumbar vertebrae fractures and luxations.] Estudo clínico e epidemiológico em cães com fraturas e luxações vertebrais toracolombares. Pesquisa Veterinária Brasileira 37(8):866-870. Departamento de Medicina Veterinária, Universidade Federal Rural de Pernambuco, Rua Dom Manoel de Medeiros s/n, Recife, PE 52171-900, Brazil. E-mail: bmaraujo85@hotmail.com

Vertebral fractures and luxations (VFL) are one of the most common and severe neurological issues found in clinical practice, with an elevated risk of permanent damage to the spinal cord. Our objective was to study the characteristics and implications associated with thoracolumbar VFL in 37 dogs, aiming assist a better clinical approach to patients with this disease. The VFL were more common in intact males with access to the street. Regarding etiology, 32 dogs were involved in car accidents. Of the dogs included in this research, 14 had non-neurological issues in other systems. The presence of unstable fractures was the most common radiographic change. In $16.2 \%$ of the dogs, vertebral lesions were observed outside the main site. Mean time until initial neurologic exam varied between one and 720 days, and most dogs were referred without external coaptation. There was a significant difference in recovery of the dogs when comparing a degree of dislocation of 0 to $25 \%$ and 76 to $100 \%$.
\end{abstract}

INDEX TERMS: Dogs, vertebra fracture, vertebra luxation, vertebral column, spinal trauma, paraplegia.

RESUMO.- Fraturas e luxações vertebrais (FLV) estão dentre as principais afecções que acometem a coluna vertebral em cães, com elevado risco de danos permanentes às estruturas neurais. Objetivou-se estudar as características e implicações associadas às FLV toracolombares em 37 cães, visando auxiliar uma melhor abordagem clínica em pacientes com esta afecção. As FLV foram mais comuns em machos não castrados com acesso a rua. Dentre a etiologia, 32 cães foram acometidos por acidente automobilístico. 14 cães apresentaram lesões não neurológicas em outros sistemas orgânicos. A presença de fraturas instáveis foi a alteração radiográfica mais comum. Em 16,2\% dos animais, observaram-se lesões vertebrais fora do foco principal. 0 tempo até $\mathrm{o}$ atendimento neurológico inicial variou de um a 720 dias, no qual a maioria foi encaminhada sem imobiliza-

\footnotetext{
${ }^{1}$ Recebido em 8 de dezembro de 2015.

Aceito para publicação em 9 de dezembro de 2016.

${ }^{2}$ Departamento de Medicina Veterinária, Universidade Federal Rural de Pernambuco, Rua Dom Manoel de Medeiros s/n, Recife, PE 52171-900, Brasil. Pesquisa de mestrado com apoio CNPq. *Autor para correspondência: bmaraujo85@hotmail.com
}

ção externa. Houve diferença estatisticamente significante quanto à recuperação neurológica ao se comparar os graus de deslocamento de 0 a $25 \%$ e de 76 a $100 \%$.

TERMOS DE INDEXAÇÃO: Cães, fratura vertebral, luxação vertebral, coluna vertebral, trauma medular, paraplegia.

\section{INTRODUÇÃO}

Fraturas e luxações vertebrais (FLV) são causadas por lesões traumáticas ou patológicas da coluna vertebral, resultando em compressão, laceração, concussão e/ou secção das estruturas neurais (Dewey 2014). É considerada uma afecção grave, devido ao elevado risco de danos medulares permanentes e corresponde a aproximadamente $7 \%$ das afecções neurológicas em cães (Fluehmann et al. 2006, Weh \& Kraus 2012).

São causadas na maioria das vezes por acidentes automobilísticos. Ocorre ainda por briga entre animais, chutes, quedas, choques contra objetos parados, neoplasias ou infecções vertebrais e osteopatias nutricionais. Afeta com mais frequência a região toracolombar da coluna vertebral $\left(\mathrm{T}_{3}-\mathrm{L}_{3}\right)$ (Araújo et al. 2009, Bali et al. 2009, Weh \& Kraus 2012). 
Cerca de 40 a $83 \%$ dos pacientes com FLV apresentam lesões intercorrentes em outros sistemas (Sturges \& LeCouteur 2007, Weh \& Kraus 2012). Por isso, torna-se necessário um exame clínico completo para reconhecer essas lesões antes de se prosseguir com as investigações específicas (Dewey 2014).

O diagnóstico baseia-se na anamnese, nos sinais clínico-neurológicos agudos e na obtenção de imagens da coluna vertebral e medula espinal (Araújo et al. 2009). Os sinais clínicos variam de hiperpatia vertebral à paralisia com perda da nocicepção, dependendo da gravidade da lesão (Fluehmann et al. 2006, Weh \& Kraus 2012, Dewey 2014). 0 tratamento conservativo consiste na administração de neuroprotetores, analgésicos e imobilização da coluna vertebral (McKee 1990, Bruce et al. 2008). 0 tratamento cirúrgico objetiva descompressão da medula espinal, alinhamento do canal vertebral, estabilização da coluna vertebral e remoção de possíveis fragmentos ósseos de dentro do canal vertebral (Bruce et al. 2008, Jeffery 2010, Cabassu 2012, Weh \& Kraus 2012).

0 prognóstico depende principalmente da avaliação da nocicepção, que indica a gravidade da lesão medular, no qual a sua presença indica prognóstico favorável e a ausência, prognóstico reservado à desfavorável (Araújo et al. 2009, Weh \& Kraus 2012).

Objetivou-se desenvolver um estudo epidemiológico de cães com fraturas e luxações vertebrais toracolombares, analisando-se as características e implicações associadas, visando auxiliar uma melhor abordagem clinica em pacientes com esta afecção.

\section{MATERIAL E MÉTODOS}

Para o experimento (Licença № 023/2012 da Comissão de Ética no Uso de Animais da Universidade Federal Rural de Pernambuco), foram utilizados 37 animais da espécie canina, durante o período entre março de 2011 e julho de 2012, sem distinção de sexo, raça ou idade, atendidos no Hospital Veterinário Universitário da Universidade Federal Rural de Pernambuco, que apresentaram fraturas e luxações vertebrais toracolombares, diagnosticadas por meio de exames neurológico e radiográfico.

Após a anamnese, foi realizada avaliação clínica geral, verificando o estado orgânico e possíveis lesões não neurológicas concomitantes. Na ausência ou após o tratamento destas, foi realizado exame neurológico para se obter o diagnóstico neuroanatômico e a gravidade da lesão. Em seguida, foram solicitados exames complementares, de acordo com a necessidade de cada caso. 0 grau de disfunção neurológica foi adaptado dos parâmetros utilizados por Bruce et al. (2008), sendo os grupos I à IV formados pelos animais que mantiveram a nocicepção intacta e o grupo $\mathrm{V}$, pelos animais que a perderam.

Inicialmente, os pacientes com lesões recentes (menos de 30 dias) foram imobilizados, a fim de se evitar lesões adicionais à medula espinal e foram medicados com meloxicam $(0,1 \mathrm{mg} / \mathrm{kg}$, via oral [VO], SID [uma vez ao dia], por sete dias), cloridrato de tramadol (4mg/kg, Vo, três vezes ao dia, por 5 dias) e omeprazol $(0,7 \mathrm{mg} / \mathrm{kg}, \mathrm{VO}, \mathrm{SID}$, por 10 dias $)$, sendo em seguida submetidos ao tratamento conservativo ou cirúrgico, de acordo com a necessidade de cada caso. Os casos crônicos (mais de 30 dias) receberam medicações e imobilização externa de acordo com a necessidade de cada caso (de acordo com a presença de instabilidade vertebral e compressão medular).
Foram considerados pertencentes ao grupo de tratamento conservativo aqueles animais que apresentaram déficits neurológicos leves (graus I, II e III), lesões estáveis, fraturas não deslocadas, com ausência ou discreta compressão medular e cães em outros graus que não se apresentaram em condições clínicas de serem submetidos a um procedimento cirúrgico ou cujos proprietários não apresentavam condições financeiras de arcar com os custos do procedimento.

Foram considerados pacientes cirúrgicos aqueles com condições orgânicas de serem submetidos ao procedimento cirúrgico e anestésico, que apresentaram déficits neurológicos graves (graus IV e V), lesões instáveis, fraturas deslocadas, evidências de compressão medular e animais que apresentaram piora ante o tratamento conservativo.

Os procedimentos cirúrgicos foram realizados no menor intervalo de tempo possível após o atendimento inicial. A fim de padronizá-los, estes foram realizadas pelos mesmos membros da equipe, efetuando-se descompressão, redução e estabilização vertebral (quando necessário), utilizando-se as técnicas descritas na literatura.

Mediante dados coletados das fichas de avaliação individual, foram analisadas as informações referentes à epidemiologia, sinais clínicos, neurológicos e exames complementares.

Em relação à epidemiologia, foram analisados os dados e frequências referentes ao sexo, raça, idade, moradia e etiologia da lesão. Para a moradia, utilizaram-se os parâmetros de Mendes \& Arias (2012) que classificaram os animais como domiciliados, semi-domiciliados e não domiciliados.

Os dados referentes aos sinais clínicos, neurológicos e radiográficos foram relacionados à forma de encaminhamento dos animais ao serviço de neurologia (com ou sem imobilização externa), ao tempo desde o momento do trauma ate o atendimento inicial; à presença de lesões não neurológicas concomitantes; aos graus de instabilidade vertebral determinada por meio do método dos três compartimentos (as fraturas foram consideradas instáveis quando dois ou três compartimentos estavam comprometidos e estáveis, quando envolviam apenas um deles) e quais foram os mais comumente envolvidos; à localização segmentar mais frequente; aos graus de acometimento neurológico e a relação com a taxa de recuperação.

Foi realizada ainda a correlação do grau de deslocamento do canal vertebral (de acordo com o método descrito por Bali et al. [2009]) com as taxas de recuperação por meio do teste não paramétrico exato de Fischer utilizando o software grapfPad InStat. versão 3.05 com nível de significância de 5\%.

\section{RESULTADOS E DISCUSSÃO}

Os dados da etiologia da lesão, localização segmentar, grau de disfunção neurológica, presença de lesões concomitantes, grau de deslocamento vertebral, tipo de tratamento e o resultado da terapia de 37 cães com fraturas e luxações vertebrais estão expostos no Quadro 1.

Dos animais incluídos na pesquisa, 21/37 animais (56,7\%) eram machos (todos não castrados). Em relação à raça, $21(56,7 \%)$ não tinham raça definida e $16(44,4 \%)$ eram de raça. Em relação aos últimos, 7/16 eram da raça Poodle, 6/16 Miniaturas pinschers, 2/16 Daschunds e um Husky siberiano (Fig.1). A idade variou de quatro meses a 20 anos, com idade média de 5,3 \pm 5 anos. Não foram computados para este parâmetro os dados de 15 animais não domiciliados, no qual os proprietários não sabiam informar a idade exata dos cães. 
Quadro 1. Etiologia da lesão, localização segmentar, grau de disfunção neurológica, presença de lesões concomitantes, grau de deslocamento vertebral, tipo de tratamento e o resultado da terapia dos 37 cães acometidos por FLV toracolombares

\begin{tabular}{|c|c|c|c|c|c|c|c|}
\hline $\begin{array}{l}\text { Grau de disfunção } \\
\text { neurológica }\end{array}$ & animal & $\begin{array}{l}\text { Etiologia } \\
\text { da lesão }\end{array}$ & $\begin{array}{c}\text { Local } \\
\text { da lesão }\end{array}$ & $\begin{array}{c}\text { Lesões em } \\
\text { outros sistemas }\end{array}$ & $\begin{array}{c}\text { Deslocamento } \\
\text { vertebral }\end{array}$ & $\begin{array}{c}\text { Tipo de } \\
\text { tratamento }\end{array}$ & $\begin{array}{l}\text { Resultado da } \\
\text { terapia }\end{array}$ \\
\hline I & 34 & Queda & $\mathrm{T}_{12}-\mathrm{T}_{13}$ & Sim & $5 \%$ & Conservativo & Recuperação total \\
\hline II & 6 & Queda & $\mathrm{T}_{1}-\mathrm{T}_{2}$ & Sim & $15 \%$ & Conservativo & Recuperação total \\
\hline II & 7 & AA & $\mathrm{L}_{3}-\mathrm{L}_{4}^{2}$ & Não & $20 \%$ & Conservativo & Recuperação total \\
\hline II & 33 & $\mathrm{AA}$ & $\mathrm{L}_{3}-\mathrm{L}_{4}$ & Não & $60 \%$ & Conservativo & Recuperação total \\
\hline III & 5 & $\mathrm{AA}$ & $\mathrm{T}_{12}-\mathrm{T}_{13}$ & Não & $10 \%$ & Conservativo & Recuperação total \\
\hline III & 8 & $\mathrm{AA}$ & $\mathrm{T}_{11}-\mathrm{T}_{12}$ & Sim & $100 \%$ & Conservativo & Recuperação total \\
\hline III & 12 & AA & $\mathrm{T}_{9}-\mathrm{T}_{10}^{12}$ & Sim & $20 \%$ & Conservativo & Recuperação total \\
\hline III & 15 & $\mathrm{AA}$ & $\mathrm{T}_{12}-\mathrm{T}_{13}$ & Sim & $15 \%$ & Conservativo & Recuperação total \\
\hline III & 21 & $\mathrm{AA}$ & $\mathrm{L}_{2}-\mathrm{L}_{3}$ & Não & $10 \%$ & Cirúrgico & Recuperação total \\
\hline III & 24 & AA & $\mathrm{T}_{11}^{2}-\mathrm{T}_{12}^{3}$ & Não & $40 \%$ & Conservativo & Recuperação total \\
\hline IV & 4 & $\mathrm{AA}$ & $\mathrm{T}_{12}-\mathrm{T}_{13}$ & Não & $100 \%$ & Cirúrgico & Recuperação total \\
\hline IV & 11 & $\mathrm{AA}$ & $\mathrm{T}_{12}-\mathrm{T}_{13}$ & Sim & $0 \%$ & Cirúrgico & $\begin{array}{l}\text { Recuperação parcial } \\
\text { (morte por IRA) }\end{array}$ \\
\hline IV & 20 & $\mathrm{AA}$ & $\mathrm{T}_{11}-\mathrm{T}_{12}$ & Não & $10 \%$ & Cirúrgico & Recuperação total \\
\hline IV & 29 & $\mathrm{AA}$ & $\mathrm{L}_{1}-\mathrm{L}_{2}$ & Não & $15 \%$ & Cirúrgico & Recuperação total \\
\hline $\mathrm{V}$ & 1 & $\mathrm{AA}$ & $\mathrm{T}_{7}-\mathrm{T}_{8}$ & Sim & $>100 \%$ & Conservativo & Não recuperou \\
\hline $\mathrm{V}$ & 2 & AA & $\mathrm{L}_{3}-\mathrm{L}_{4}{ }^{\circ}$ & Não & $90 \%$ & Conservativo & $\begin{array}{l}\text { Não recuperou } \\
\text { (eutanásia) }\end{array}$ \\
\hline V & 3 & $\mathrm{AA}$ & $\mathrm{T}_{7}-\mathrm{T}_{8}$ & Não & $10 \%$ & Conservativo & $\begin{array}{l}\text { Não recuperou } \\
\text { (eutanásia) }\end{array}$ \\
\hline $\mathrm{V}$ & 9 & AA & $\mathrm{T}_{11}-\mathrm{T}_{12}$ & Não & $50 \%$ & Conservativo & $\begin{array}{l}\text { Não recuperou } \\
\text { (eutanásia) }\end{array}$ \\
\hline V & 10 & $\mathrm{AA}$ & $\mathrm{T}_{12}-\mathrm{T}_{13}$ & Não & $100 \%$ & Conservativo & Não recuperou \\
\hline $\mathrm{V}$ & 13 & $\mathrm{AA}$ & $\mathrm{T}_{12}-\mathrm{T}_{13}$ & Não & $20 \%$ & Conservativo & $\begin{array}{l}\text { Não recuperou } \\
\text { (eutanásia) }\end{array}$ \\
\hline V & 14 & $\mathrm{AA}$ & $\mathrm{T}_{12}-\mathrm{T}_{13}$ & Não & $100 \%$ & Cirúrgico & Não recuperou \\
\hline $\mathrm{V}$ & 16 & Queda & $\mathrm{T}_{12}-\mathrm{T}_{13}$ & Não & $100 \%$ & - & Eutanásia sem tratamento \\
\hline $\mathrm{V}$ & 17 & Queda & $\mathrm{L}_{3}-\mathrm{L}_{4}$ & Sim & $5 \%$ & Conservativo & Não recuperou \\
\hline V & 18 & $\mathrm{AA}$ & $\mathrm{T}_{13}-\mathrm{l}_{1}$ & Sim & $90 \%$ & - & $\begin{array}{c}\text { Óbito (ruptura } \\
\text { de vesícula urinária) }\end{array}$ \\
\hline V & 19 & Queda & $\mathrm{T}_{11}-\mathrm{T}_{12}$ & Não & $30 \%$ & Cirúrgico & Não recuperou \\
\hline V & 22 & $\begin{array}{l}\text { Agressão de } \\
\text { outro cão }\end{array}$ & $\mathrm{T}_{12}-\mathrm{T}_{13}$ & Sim & $10 \%$ & - & $\begin{array}{c}\text { Morte } \\
\text { (trauma torácico) }\end{array}$ \\
\hline V & 23 & $\mathrm{AA}$ & $\mathrm{T}_{13}-\mathrm{L}_{1}$ & Não & $95 \%$ & Cirúrgico & Não recuperou \\
\hline V & 25 & $\mathrm{AA}$ & $\mathrm{T}_{12}-\mathrm{T}_{13}$ & Não & $100 \%$ & Cirúrgico & Não recuperou \\
\hline V & 26 & $\mathrm{AA}$ & $\mathrm{T}_{11}-\mathrm{T}_{12}$ & Não & $100 \%$ & Conservativo & Não recuperou \\
\hline V & 27 & $\mathrm{AA}$ & $\mathrm{T}_{11}-\mathrm{T}_{12}$ & Não & $>100 \%$ & Cirúrgico & Não recuperou \\
\hline V & 28 & $\mathrm{AA}$ & $\mathrm{T}_{9}-\mathrm{T}_{10}$ & Não & $80 \%$ & Conservativo & Não recuperou \\
\hline V & 30 & $\mathrm{AA}$ & $\mathrm{T}_{13}-\mathrm{L}_{1}$ & Não & $30 \%$ & Conservativo & Não recuperou \\
\hline V & 31 & AA & $\mathrm{T}_{12}-\mathrm{T}_{13}$ & Sim & $100 \%$ & Cirúrgico & Não recuperou \\
\hline V & 32 & $\mathrm{AA}$ & $\mathrm{L}_{1}-\mathrm{L}_{2}$ & Sim & $20 \%$ & Conservativo & Não recuperou \\
\hline V & 35 & $\mathrm{AA}$ & $\mathrm{T}_{11}-\mathrm{T}_{12}$ & Sim & $100 \%$ & Cirúrgico & Não recuperou \\
\hline V & 36 & $\mathrm{AA}$ & $\mathrm{T}_{1}-\mathrm{T}_{12}$ & Não & $100 \%$ & Cirúrgico & Não recuperou \\
\hline $\mathrm{V}$ & 37 & $\mathrm{AA}$ & $\mathrm{T}_{13}-\mathrm{L}_{1}$ & Sim & $>100 \%$ & Cirúrgico & Não recuperou \\
\hline
\end{tabular}

$\mathrm{AA}=$ Acidente automobilístico, IRA = Insuficiência renal aguda.

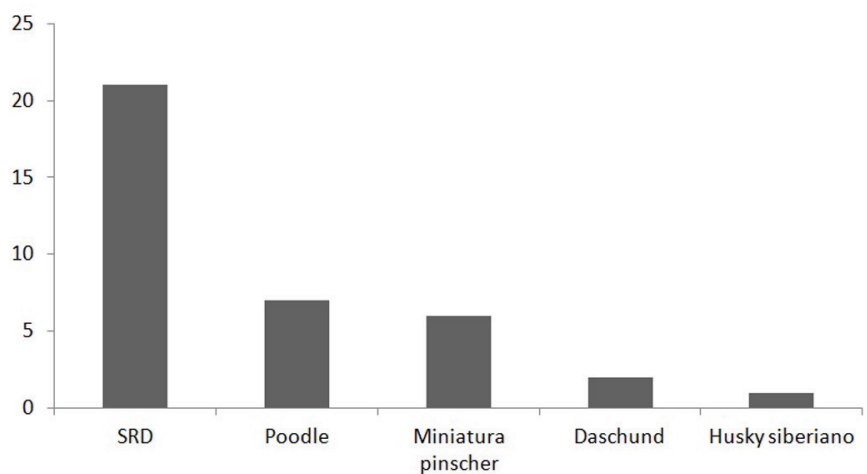

Fig.1. Distribuição dos animais acometidos por fraturas e luxações vertebrais em relação à raça.

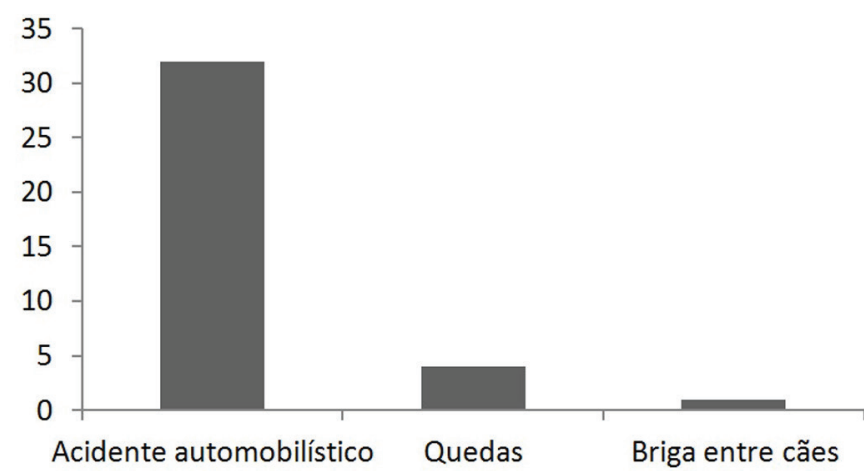

Fig.2. Distribuição da etiologia da lesão vertebral em 37 cães com fraturas e luxações vertebrais. 
Em relação à moradia, 19/37 animais (51,3\%) eram domiciliados. Destes, 13 cães $(68,4 \%)$ tinham livre acesso a rua e seis $(31,5 \%)$ tinham acesso restrito. Três animais $(8,1 \%)$ eram semi-domiciliados e 15 animais $(40,5 \%)$ não domiciliados. Os resultados deste estudo assemelham-se com os de Mendes \& Arias (2012) no qual dos 48 cães acometidos por fraturas e luxações vertebrais, 37 cães eram domiciliados, um semi-domiciliado e 10 eram não domiciliados.

Dentre a etiologia da lesão, 32 animais $(86,4 \%)$ foram acometidos por acidente automobilístico; quatro animais $(10,8 \%)$ por queda e um animal $(2,7 \%)$ por ataque de outro cão (Fig.2), corroborando esse predomínio com McKee (1990), Fluehmann et al. (2006), Lorenz et al. (2011) e Dewey (2014). Desta forma, Mendes \& Arias (2012) recomendam que os médicos veterinários orientem os proprietários de animais quanto à adoção de medidas de segurança que minimizem tais riscos.

As fraturas e luxações vertebrais foram mais comuns em machos não castrados com acesso a rua, que foram envolvidos em acidentes automobilísticos. Para Turner (1987), o aumento na incidência dessas lesões em cães machos não castrados pode ser devido ao comportamento de passeio, tornando-os mais susceptíveis a acidentes automobilísticos. Os resultados foram similares aos descritos por Turner (1987), Bruce et al. (2008) e Mendes \& Arias (2012) que observaram maior frequência em cães machos e jovens, provavelmente devido ao comportamento reprodutivo destes animais.

De acordo com Fighera et al. (2008), a alta prevalência do traumatismo espino-medular em cães atropelados provavelmente se deve ao local do impacto do veículo no corpo dos mesmos, pois a maioria são atingidos lateralmente. Complementam ainda que a altura média dos para-choques da maior parte dos veículos é semelhante à altura média da coluna vertebral de cães de porte médio e grande. Seguindo o mesmo raciocínio, os autores desta pesquisa propõem que, em cães de pequeno porte (oito com peso inferior a 4 $\mathrm{kg}$ ), o principal mecanismo de lesão medular pode ser por rolamento do animal sob o veículo automotivo, ocasionando torção da coluna vertebral, gerando fratura vertebral obliqua, o qual ocorreu em $62,5 \%$ dos animais deste trabalho.

Dos 37 animais, apenas dois $(5,4 \%)$ foram encaminhados ao atendimento neurológico com imobilização vertebral externa. Um não possuía nocicepção (grau V de disfunção neurológica) e o outro a manteve (grau III) no atendimento inicial. 0 primeiro animal não apresentou recuperação funcional e o segundo apresentou recuperação total. De acordo com Olby (1999) e Weh \& Kraus (2012), os cães com suspeita de FLV devem receber imobilização temporária no transporte e enquanto se decide pelo tratamento adequado, no intuito de evitar a ocorrência de traumatismos adicionais à coluna vertebral e a medula espinal. De acordo com Weh \& Kraus (2012), a lesão medular é dinâmica e pode piorar caso a imobilização vertebral externa seja realizada tardiamente.

O tempo desde a ocorrência do trauma ate o atendimento neurológico inicial variou de 1 a 720 dias, com tempo médio de $35 \pm 120$ dias. 0 tempo para atendimento dos casos de FLV foi superior ao de outros trabalhos descritos na literatura (McKee 1990, Bali et al. 2009, Mendes \& Arias 2012) e refletiu a falta de encaminhamento desses animais aos centros especializados em tempo hábil, nesta região.

Quatorze $(37,8 \%)$ animais apresentaram lesões não neurológicas em outros sistemas, decorrentes do trauma que originou a FLV. Destes animais, 10/14 (71,4\%) foram acometidos por acidentes automobilísticos. Em relação ao tipo de lesão, quatro apresentavam fratura femoral, três foram acometidos por trauma torácico, dois por fratura pélvica, dois por fratura rádio-ulnar, um animal por ruptura da vesícula urinária, um por desluvamento naso-labial, um por luxação coxofemoral bilateral e um por eventração abdominal, sendo ainda que um deles apresentou duas lesões concomitantemente. Dois $(14,2 \%)$ foram a óbito em decorrência destas complicações (ruptura de vesícula urinária e trauma torácico).

Este resultado assemelha-se aos encontrados por Sturges \& LeCouteur (2007) e Weh \& Kraus (2012), quando mencionaram que cerca de 40 a $83 \%$ dos casos de fraturas e luxações da coluna toracolombar ocorrem com problemas concomitantes em outros sistemas. De acordo com Dewey (2014), pacientes com FLV frequentemente apresentam lesões intercorrentes, sendo de extrema importância que essas outras lesões, que podem não ser imediatamente aparentes, não sejam negligenciadas em favor do tratamento de lesões espinais óbvias (Jeffery 2010).

Em relação ao grau de instabilidade vertebral, 33 cães $(89,1 \%)$ apresentaram fraturas instáveis e quatro $(10,8 \%)$ fraturas estáveis. Das fraturas instáveis, 30 apresentaram os três compartimentos envolvidos e três apresentaram dois compartimentos acometidos. Nos quatro animais com fratura estável, o único compartimento envolvido foi o ventral, sendo esse parâmetro de fundamental importância na determinação do tipo de tratamento (conservativo ou cirúrgico) na presente investigação.

Observou-se predominância das lesões vertebrais localizadas no segmento $\mathrm{T}_{12}-\mathrm{T}_{13}$ da coluna vertebral $(32,4 \%)$, seguido dos segmentos $\mathrm{T}_{11}-\mathrm{T}_{12}(21,6 \%), \mathrm{T}_{13}-\mathrm{L}_{1}(10,8 \%)$ e $\mathrm{L}_{3}-\mathrm{L}_{4}(8,1 \%)$ (Fig.3). Estes resultados corroboraram com os relatos de Jeffery (2010), quando mencionou que a junção toracolombar $\left(\mathrm{T}_{10}-\mathrm{L}_{2}\right)$ é um local muito comum de FLV, constituindo aproximadamente $50 \%$ de todas as fraturas de coluna vertebral em cães. Cabassu (2012) relatou que o segmento $\mathrm{T}_{12}-\mathrm{T}_{13}$ é um dos três mais móveis da coluna

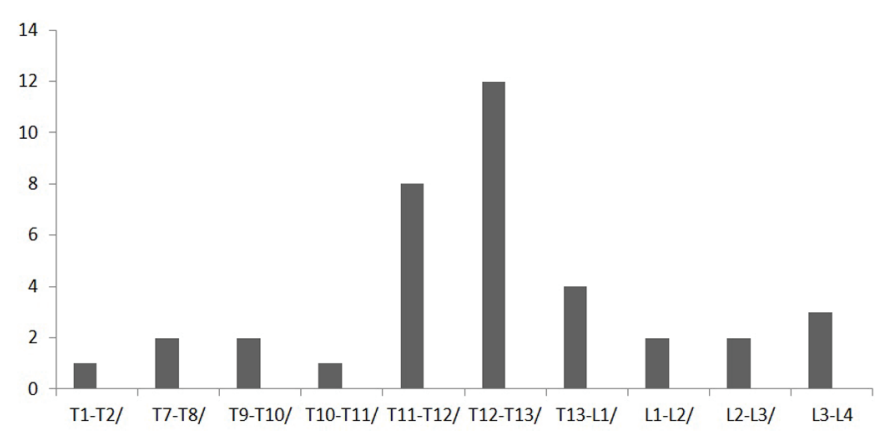

Fig.3. Distribuição da localização segmentar da lesão em 37 cães com fraturas e luxações vertebrais toracolombares. 
vertebral e, portanto, mais sensível às forças externas. Esta localização na coluna toracolombar é mais susceptível a ocasionar lesão medular grave devido à estrita relação da coluna vertebral com a medula espinal (Weh \& Kraus 2012).

Em relação ao grau de disfunção neurológica e as taxas de recuperação, $14 / 37$ cães $(37,8 \%)$ tinham nocicepção intacta (graus I a IV), no qual independente do tratamento conservativo ou cirúrgico, a taxa de recuperação da deambulação voluntária e funções viscerais foi de $100 \%$. Enquanto que $23 / 37$ animais $(62,1 \%)$ perderam a nocicepção (grau V), no qual nenhum recuperou a deambulação voluntária, independente do tipo de tratamento. 0 prognóstico desfavorável de cães com FLV que perdem a nocicepção pode ser justificado pelo fato desta perda estar relacionado à grave lesão da medula espinal (Weh \& Kraus 2012). Desta forma, o parâmetro de ausência ou presença de nocicepção foi um confiante indicativo do prognóstico em cães com fraturas e luxações vertebrais, conforme relatado por alguns autores (Araújo et al. 2009, Bali et al. 2009, Jeffery 2010, Weh \& Kraus 2012).

Analisando o grau de deslocamento vertebral no exame radiográfico, com o grau de disfunção neurológica e o resultado da terapia, foi possível observar que a maior parte dos cães estava incluída nos graus de deslocamento de 0 a $25 \%$ e de 76 a $100 \%$, sendo que a maioria dos animais incluídos no grupo 0 - 25\% possuía nocicepção e recuperou as funções neurológicas. Enquanto que a maioria dos animais do grupo de $76-100 \%$ perdeu a nocicepção e não se recuperou. Esta discrepância apresentou diferença estatisticamente significante ao se comparar esses dois graus de deslocamento ( 0 a $25 \%$ e de 76 a $100 \%$ ), concluindo que este parâmetro realmente tem importância na avaliação do prognóstico de cães com FLV toracolombares. No entanto, o grau de deslocamento do canal vertebral não deve ser o parâmetro mais importante para de determinar o prognóstico, uma vez que houve animais com deslocamento vertebral de $100 \%$ que recuperaram e animais com desníveis oscilando entre cinco a10\% que não se recuperaram.

Segundo Vilela et al. (2007), a avaliação radiográfica não substitui o exame neurológico, pois as radiografias podem não demonstrar o deslocamento vertebral máximo no momento da lesão (Dewey 2014). Desta forma, deve-se avaliar principalmente a nocicepção, que é o principal parâmetro para indicar a gravidade da lesão medular (Araújo et al. 2009, DeLahunta \& Glass 2009, Weh \& Kraus 2012).

\section{CONCLUSÕES}

As fraturas e luxações vertebrais nos cães apresentam riscos de lesões medulares irreversíveis e intercorrentes em outros sistemas.

Tem ocorrência mais comum em machos não castrados com acesso a rua, acometidos por acidente automobi- lístico, sendo, portanto, uma afecção passível de prevenção.

Torna-se ainda necessário a conscientização de alguns profissionais e proprietários para encaminharem esses pacientes a centros especializados em tempo hábil.

0 parâmetro de deslocamento vertebral foi eficiente para predizer o prognóstico de cães acometidos por FLV. No entanto, não deve ser o dado mais importante para de determinar o prognóstico de animais acometidos por FLV, prevalecendo para tal a presença ou ausência de nocicepção.

\section{REFERÊNCIAS}

Araújo B.M., Arias M.V.B. \& Tudury E.A. 2009. Paraplegia aguda com perda da percepção de dor profunda em cães: revisão de literatura. Clín. Vet. 14(81):70-82.

Bali M.S., Lang J., Jaggy A., Spreng D., Doherr M.G. \& Forterre F. 2009. Comparative study of vertebral fractures and luxations in dogs and cats. Vet. Comp. Orthop. Traumatol. 22(3):47-53

Bruce C.W., Brisson B.A. \& Gyselinck K. 2008. Spinal fractures in dogs and cats: a retrospective evaluation of 95 cases. Vet. Comp. Orthop. Traumatol. 21(3):280-284.

Cabassu J.P. 2012. Treatment of thoracic and lumbar vertebral fractures, p.129-131. In: Ibid. (Ed.), Proc. European Society of Veterinary Orthopaedics and Traumatology (ESVOT), Bologna. (Abstract)

DeLahunta A., Glass E. \& Kent M. 2015. Veterinary Neuroanatomy and Clinical Neurology. 4th ed. Elsevier, Philadelphia. 587p.

Dewey C.W. 2014. Cirurgia da coluna toracolombar, p.1508-1528. In: Fossum T.W. (Ed), Cirurgia de Pequenos Animais. $4^{\underline{a}}$ ed. Elsevier, Rio de Janeiro.

Fighera R.A., Silva M.C., Souza T.M., Brum J.S., Kommers D., Graça D.L., Irigoyen L.F. \& Barros C.S.L. 2008. Aspectos patológicos de 155 casos fatais de cães atropelados por veículos automotivos. Ciência Rural 38(5):1375-1380.

Fluehmann G., Doherr M.G. \& Jaggy A. 2006. Canine neurological diseases in a referral hospital population between 1989 and 2000 in Switzerland. J. Small. Anim. Pract. 47(10):582-587

Jeffery N.D. 2010. Vertebral fracture and luxation in small animals. Vet. Clin. N. Am., Small. Anim. Pract. 40(5):809-828.

Lorenz M.D., Coates J.R. \& Kent M. 2011. Handbook of Veterinary Neurology. $5^{\text {th }}$ ed. Elsevier, St Louis. 545p.

McKee W.M. 1990. Spinal trauma in dogs and cats: a review of 51 cases. Vet. Rec. 126(6):285-289.

Mendes D.S. \& Arias M.V.B. 2012. Traumatismo da medula espinhal em cães e gatos: estudo prospectivo de 57 casos. Pesq. Vet. Bras. 32(12):13041312.

Olby N. 1999. Current concepts in the management of acute spinal cord injury. J. Vet. Intern. Med. 13(5):399-407.

Sturges B.K. \& LeCouteur R.A. 2007. Fraturas e luxações vertebrais, p.1244-1260. In: Slatter D. (Ed.), Manual de Cirurgia de Pequenos Animais. $3^{a}$ ed. Manole, São Paulo.

Turner W.D. 1987. Fractures and fractures-luxations of the lumbar spine: a retrospective study in the dog. J. Am. Vet. Med. Assoc. 23(459):459-464.

Vilela L.M., Baiotto G.C. \& Barros S.V.S.G. 2007. Terapêutica do trauma medular agudo. Nosso Clínico 10(59):46-51.

Weh M. \& Kraus K.H. 2012. Spinal fractures and luxations, p.487-503. In: Tobias K.M. \& Johnston S.A. (Eds), Veterinary Surgery Small Animals. Saunders, Elsevier. 